\title{
TNF-Independent Development of Transient Anemia of Chronic Disease in a Mouse Model of Protracted Septic Peritonitis
}

\author{
Thomas Schubert, Bernd Echtenacher, Ferdinand Hofstädter, and \\ Daniela N. Männel \\ Department of Pathology (TS, BE, FH, DNM), University of Regensburg, Regensburg, Germany
}

\begin{abstract}
SUMMARY: TNF is considered one of the inflammatory cytokines and contributes mainly to the generation of anemia of chronic disease (ACD). In nude mice TNF has been reported to impair iron metabolism and erythropoiesis, leading to anemia with a low serum iron and preserved iron stores. In this work, we established a murine model for ACD based on sublethal cecal ligation and puncture (CLP) with ensuing protracted peritonitis. Starting on Day 3 after CLP, a severe protracted depression of erythropoiesis in the bone marrow was noted. Two weeks after CLP, we observed a moderate normochromic anemia, low serum iron concentration, and preserved iron stores consistent with transient ACD. To determine whether TNF contributes to the development of ACD in vivo, we neutralized TNF after CLP shortly before and during the phase of most severe bone marrow depression to prevent anemia. Additionally, we studied TNF-deficient mice undergoing CLP. Two weeks after CLP, we determined red blood count, hemoglobin concentration, hematocrit, serum iron concentration, and iron stores in spleens of wild-type mice, TNF-deficient mice, and mice after neutralization of TNF. Neutralization of TNF after CLP could not prevent mice from contracting anemia. Accordingly, TNF-deficient mice developed anemia to the same extent as wild-type mice. Serum iron concentration was lowered and iron stores were overloaded in both TNF-deficient and wild-type mice after CLP. Our results clearly demonstrate that TNF is not a mediator of ACD in our model with transient anemia induced by protracted septic peritonitis. (Lab Invest 2003, 83:1743-1750).
\end{abstract}

A nemia commonly occurs in chronic infection, inflammation, and malignancy (Barrett-Connor, 1972; Zucker et al, 1974). This type of anemia is characterized by decreased plasma iron and ironbinding capacity, normal or elevated iron stores, modest decrease of red cell survival, and relative failure of bone marrow to increase red cell production. It was first described with infection (Cartwright, 1966); however, because of its association with other instances of chronic disease such as chronic inflammation and cancer, it is called anemia of chronic diseases (ACD). An understanding of the role that inflammatory cytokines play in most of the diseases underlying ACD has suggested that cytokines are important factors in the pathophysiology of ACD. Inflammatory cytokines such as TNF and IL-1 have effects on iron metabolism and red cell survival in vivo (Freireich et al, 1957; Johnson et al, 1989; Moldawer et al, 1989; Rogers et al, 1990). Inflammatory cytokines have also been implicated in the inappropriately low erythropoietin (EPO) levels in ACD patients (Miller et al, 1990). Supporting the idea

DOI: 10.1097/01.LAB.0000101693.12149.2C

Received February 17, 2003.

This work was supported by a ReForM A grant provided by Regensburg University.

Address reprint requests to: Dr. Thomas Schubert, Department of Pathology, University of Regensburg, Franz-Josef-Strauss-Allee 11, 93053 Regensburg, Germany. E-mail: thomas.schubert@klinik.uni-regensburg.de that cytokines could contribute to ACD are data that suggest that TNF, IL-1, and IFN- $\gamma$ also exert direct inhibitory effects on hematopoiesis in vivo and in vitro (Means et al, 1992, 1994; Means and Krantz, 1993; Salleri et al, 1996; Wang et al, 1995). In summary, our present knowledge of the role of cytokines in ACD is mainly based on clinical correlations and experiments describing effects of several cytokines in vivo and in vitro. According to these data, TNF, IL-1, and IFN- $\gamma$ seem to be the most important inflammatory factors involved in the generation of ACD. However, the contribution of distinct cytokines to the different aspects of ACD has not been determined in vivo due to the lack of appropriate animal models for ACD.

To determine whether TNF contributes to the development of $A C D$ in vivo we established a murine model for transient ACD based on sublethal cecal ligation and puncture (CLP) with ensuing protracted peritonitis. Using this model we neutralized TNF after CLP to prevent anemia and studied TNF-deficient mice undergoing CLP.

\section{Results}

\section{Mice after CLP Develop Transient ACD within 2 Weeks}

Mice display a slight but constant decrease in hemoglobin concentration, RBC count, and hematocrit after CLP, resulting in mild anemia (Fig. 1, A to F). Anemia is most marked 2 weeks after CLP. At this time point, the volume of erythrocytes was slightly decreased and serum iron concentration was lowered (Fig. 1, D and F). The mean 

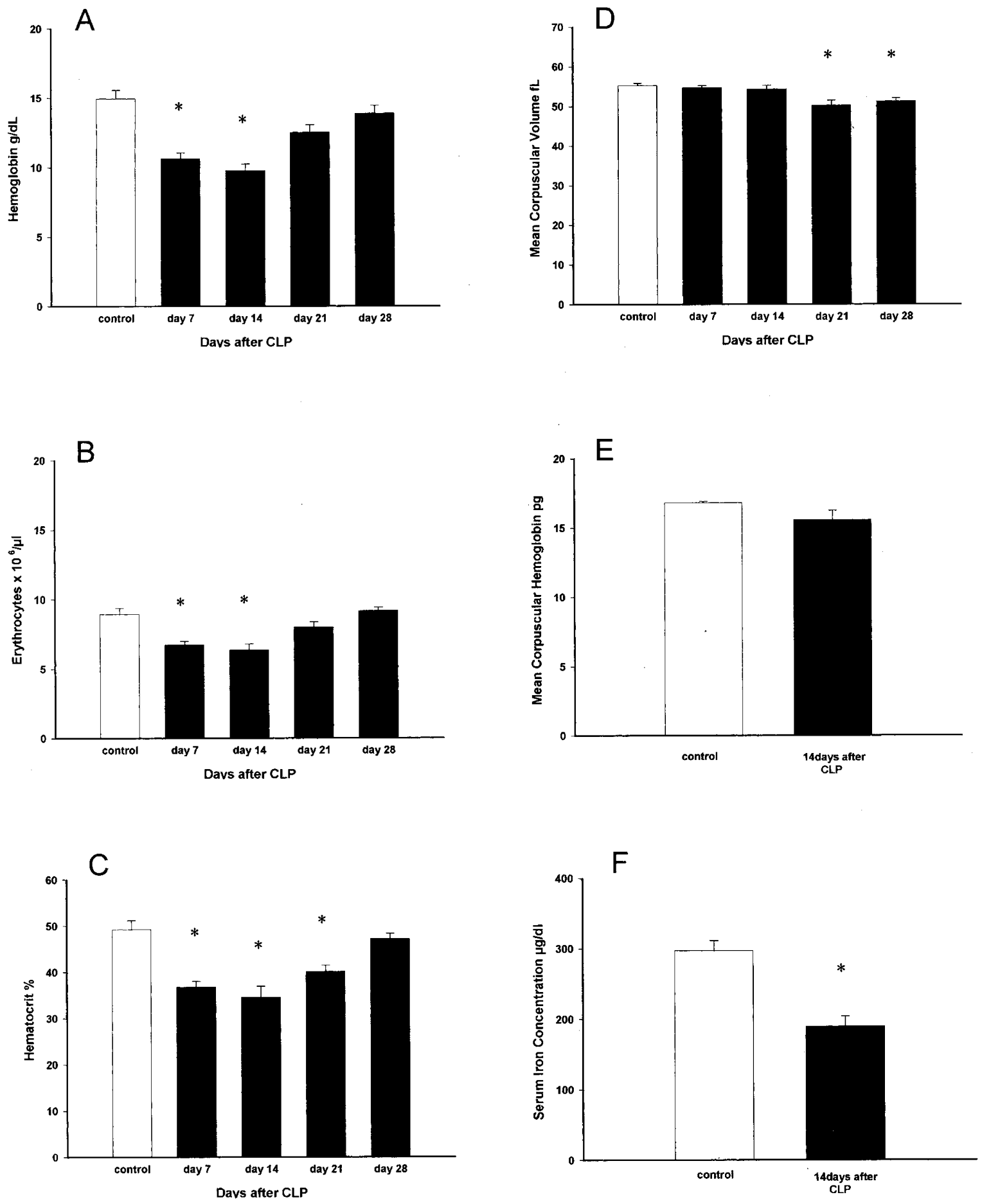

Figure 1.

Mice contract a normocytic, normochromic anemia after cecal ligation and puncture (CLP) accompanied by lowered serum iron levels. Hemoglobin concentration (A), red blood cell count $(B)$, and hematocrit $(C)$ in untreated control mice and mice after CLP were determined at the indicated time points. One, 2, and 3 weeks after CLP, a significant, moderate decrease in hemoglobin concentration (Weeks 1 and 2: $p<0.01$; Week 3: $p<0.05$ ), red blood cell count (Weeks 1 and 2: $p<0.01$ ), and hematocrit $(p<0.01)$ was found. Anemia was most marked 2 weeks after CLP. Results are given as mean \pm SD $(n=5)$. (D) Mean corpuscular erythrocyte volume (Weeks 3 and 4: $p<0.01$ ), (E) mean corpuscular hemoglobin concentration, and (F) serum iron concentration were determined 2 weeks after CLP ( $p<0.001$ ). Results are given as mean \pm SD $(n=5)$.

corpuscular volume of untreated control NMRI mice ranged from $51.1 \mathrm{fl}$ to $56.9 \mathrm{fl}$. Mean corpuscular hemoglobin concentration of erythrocytes was not changed significantly (Fig. 1D). Iron stores in spleen and liver were overloaded as revealed by staining for iron (data not shown). In summary, 2 weeks after CLP, mice suffered from mild microcytic and normochromic anemia, with lowered serum iron levels fulfilling the criteria of ACD. 


\section{ACD in Mice after CLP Is Caused by Underproduction of Bone Marrow}

Reticulocyte counts in peripheral blood rose transiently on Day 1 after CLP followed by a drop to $10 \%$ of control values on Day 3 and Day 4 (Fig. 2A). Later, extramedullary hematopoiesis in liver and spleen was triggered and reticulocyte counts rose again. Extramedullary hematopoiesis was reflected in increasing spleen weights and expansion of the red pulp starting from Day 7 on (data not shown). The number of erythropoietic cells in the bone marrow remained relatively low especially in the first week and slowly recovered during Weeks 2 and 3 after CLP (Fig. 2B). There was a slow, continuous decrease of hemoglobin concentration, RBC count, and hematocrit during the first days after CLP as revealed by daily measurements (data not shown), indicating that acute hemor-
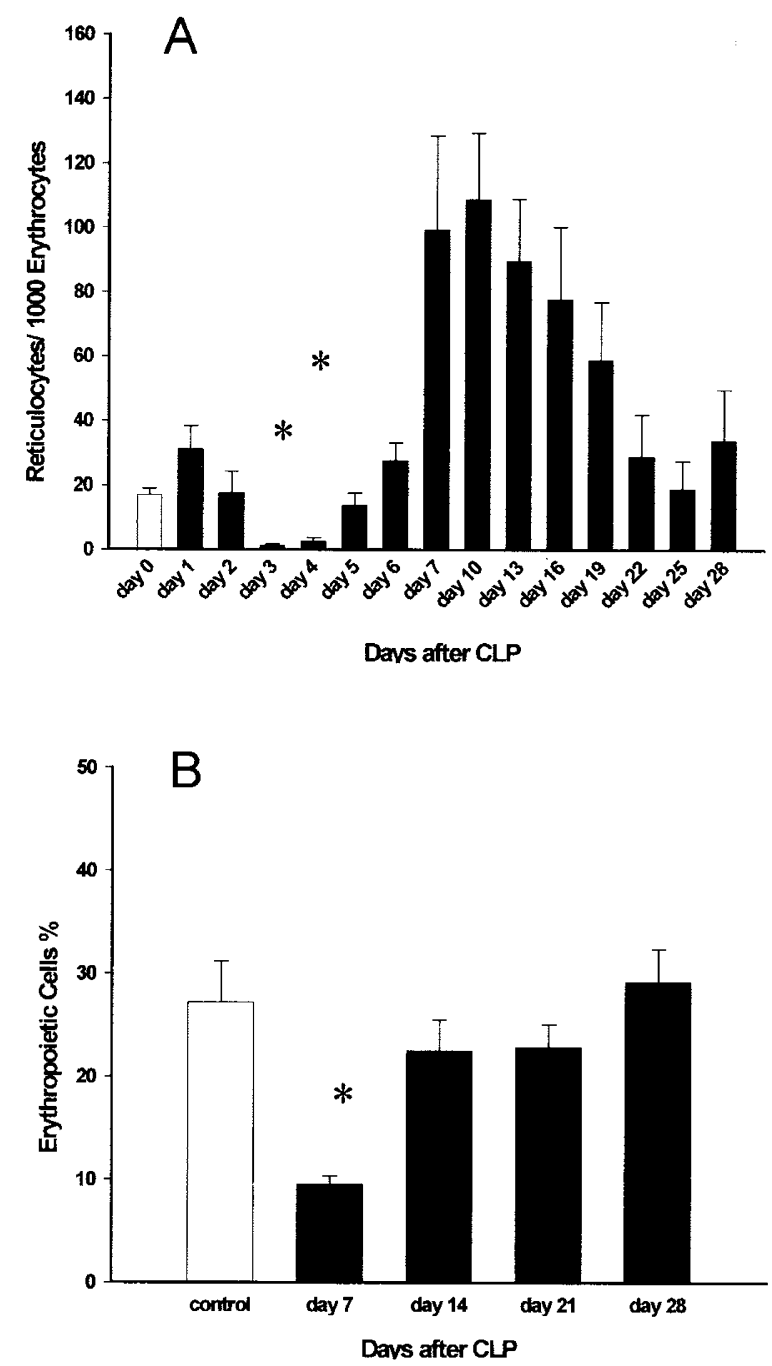

Figure 2.

Marked depression of erythropoiesis was observed in mice 1 week after cecal ligation and puncture (CLP). (A) The reticulocyte count decreased significantly on Days 3 and 4 after $\operatorname{CLP}(p<0.01, p<0.01$, respectively; $n=8)$. In the second week after CLP, increasing extramedullary hematopoiesis was reflected in rising reticulocyte counts. (B) The relative number of erythropoietic cells in bone marrow smears was decreased in the first week after CLP $(p<0.01$, $n=5)$. rhage or disseminated intravascular coagulation is not the cause of anemia.

\section{Serum-EPO Concentration Is Not Significantly Lowered during Transient ACD}

We found no significant differences in EPO serum concentrations between untreated control animals and animals 1, 2, 3, or 4 weeks after CLP (Fig. 3). The values oscillated around the control value with a trend to an increase 1 week after CLP ensued by a trend to a slight decrease 2 weeks after CLP. The rising EPO level 1 week after CLP is inversely correlated with depressed erythropoiesis in the bone marrow 1 week after CLP (Fig. 2B). The decreasing EPO level 2 weeks after CLP is inversely correlated with very high reticulocyte counts (Fig. 2A). At the same time point, erythropoiesis in the bone marrow has almost recovered (Fig. 2B). A decrease of EPO production after CLP can therefore be ruled out as cause of transient ACD.

\section{Neutralization of TNF Does Not Attenuate ACD after CLP}

To determine the role of TNF in the development of ACD, endogenous TNF was neutralized after CLP. Mice were treated with neutralizing antibodies to TNF on Day 2 after CLP. Two weeks after CLP when anemia was most severe, control-treated mice and mice treated with anti-TNF had developed anemia to the same extent as determined by hemoglobin, RBC counts, and hematocrit (Fig. $4 \mathrm{~A}$ to $\mathrm{C}$ ). Reticulocyte counts were observed for 2 weeks (Fig. 4 D). In both control-treated mice and anti-TNF-treated mice, a drop in reticulocyte counts was noted on Days 2 and 4 after CLP, indicating an identical course of depression of bone marrow erythropoiesis.

\section{TNF-Deficient Mice Develop ACD after CLP to the Same Extent as Wild-Type Mice}

To further test the role of TNF in development of ACD, TNF-deficient mice were used to study ACD after CLP

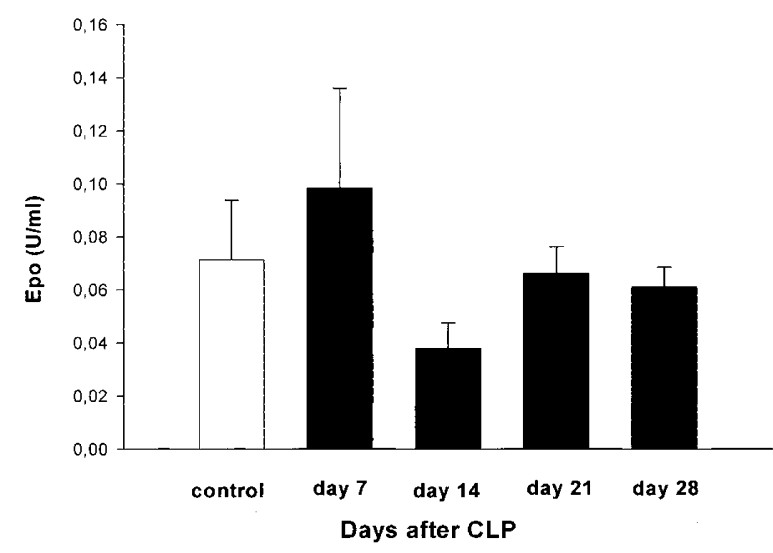

Figure 3.

Erythropoietin $(E p o)$ serum concentrations are not significantly decreased during transient anemia of chronic disease. Erythropoietin serum concentrations in untreated control mice and mice after cecal ligation and puncture $(C L P)$ were determined at the indicated time points by ELISA. Results are given as mean $\pm \mathrm{SD}(n=5)$. 

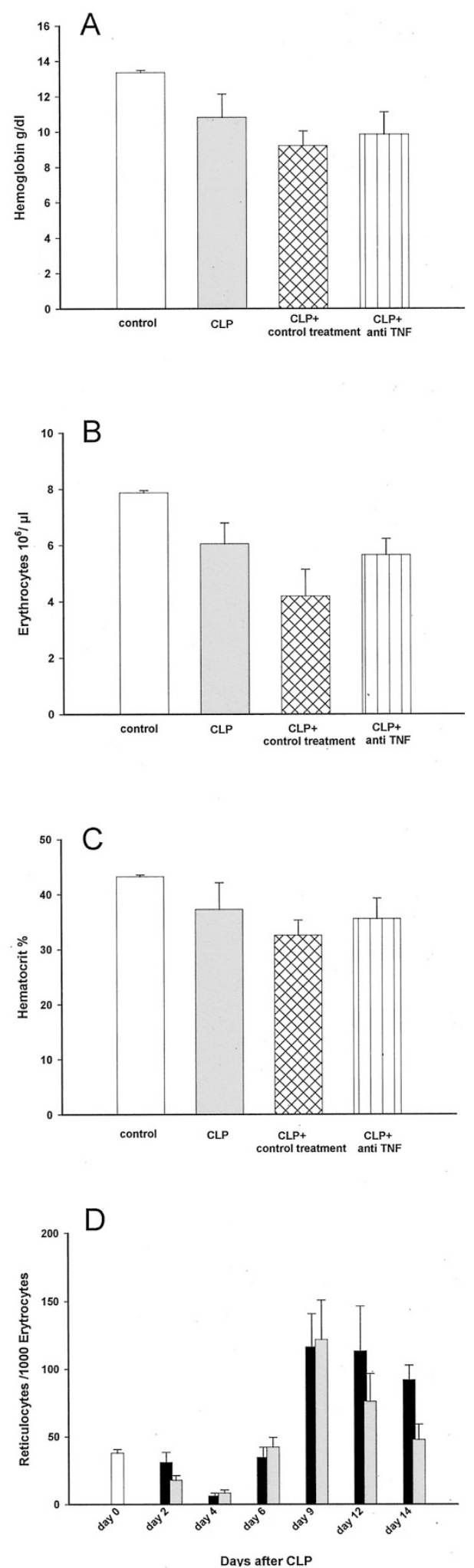

Figure 4.

Neutralization of TNF after cecal ligation and puncture (CLP) had no ameliorating effect on red blood parameters and production of erythrocytes 2 weeks after CLP. (A) Hemoglobin concentration, (B) red blood count, and (C) hematocrit in control-treated mice and anti-TNF-treated mice were equally decreased after CLP. Results are given as mean $\pm \mathrm{SD}(n=5)$. (D) The time course of reticulocyte counts in control mice and anti-TNF-treated mice after CLP was determined during 2 weeks after CLP. Results are given as the mean $\pm \mathrm{SD}(n=5)$. in the absence of TNF. Wild-type and TNF-deficient mice developed ACD to the same extent as determined by hemoglobin concentration, RBC count, and hematocrit (Fig. 5, A to C). Reticulocyte counts were observed over 2 weeks. In both wild-type mice and TNF-deficient mice, a drop in reticulocyte counts was noted on Days 3 and 4 after CLP followed by a rise on Days 7 to 9 (data not shown). Mean corpuscular volume and mean corpuscular hemoglobin concentration did not differ between TNF-deficient mice and wild-type mice 2 weeks after CLP (data not shown).

\section{Iron Metabolism in TNF-Deficient and Wild-Type Mice after CLP is Equally Disturbed}

Serum iron concentrations were moderately decreased (Fig. 6A) and iron stores in the spleen were overloaded in both TNF-deficient mice and wild-type mice 2 weeks after CLP. In untreated control mice, only few iron-positive cells were seen in the red pulp. Wild-type and TNF-deficient mice 2 weeks after CLP showed an equally increased amount of iron-positive cells in the red pulp. Some iron-positive cells were found in the white pulp (Fig. 6B).

\section{Discussion}

In the past decade, TNF has been considered as the inflammatory cytokine mainly contributing to the generation of ACD in different ways. TNF has been shown to affect the iron metabolism and erythropoiesis in several murine models.

High concentrations of TNF induced anemia with a low serum iron and preserved iron stores in nude mice (Johnson et al, 1989). Iron release from macrophages was inhibited by TNF in vitro (Alvarez-Hernandez et al, 1989). Moreover, serum levels of TNF have been shown to correlate inversely with hemoglobin concentration in patients with rheumatoid arthritis and ACD (Stockenhuber et al, 1994; Vreugdenhil et al, 1992). TNF inhibited the formation of early red cell colonies in the bone marrow both in vitro (Akahane et al, 1987; Rusten and Jacobsen, 1995) and in nude mice (Johnson et al, 1989). Studies using mice deficient for either the TNF receptors type I or type II indicated that this effect was mediated principally by the TNF receptor type I, possibly through inhibition of the cell cycle of erythroid precursor cells from the G0/G1 to the $S$ phase (Zhang et al, 1995).

The reduction in erythroid colony formation could partly be overcome in vitro by adding excess EPO to the culture system (Jongen-Lavrencic et al, 1994). EPO administration also reversed some of the suppressive effects on red cell formation in TNF-treated mice in a dose-dependent manner (Johnson et al, 1990). The reported effects of TNF on erythropoiesis and iron metabolism in vivo and in vitro supported the hypothesis that TNF besides IFN- $\gamma$ (Means et al, 1992, 1994) could be one of the major mediators of ACD. However, due to the lack of well-characterized in vivo models for ACD it has not been proven that up- 

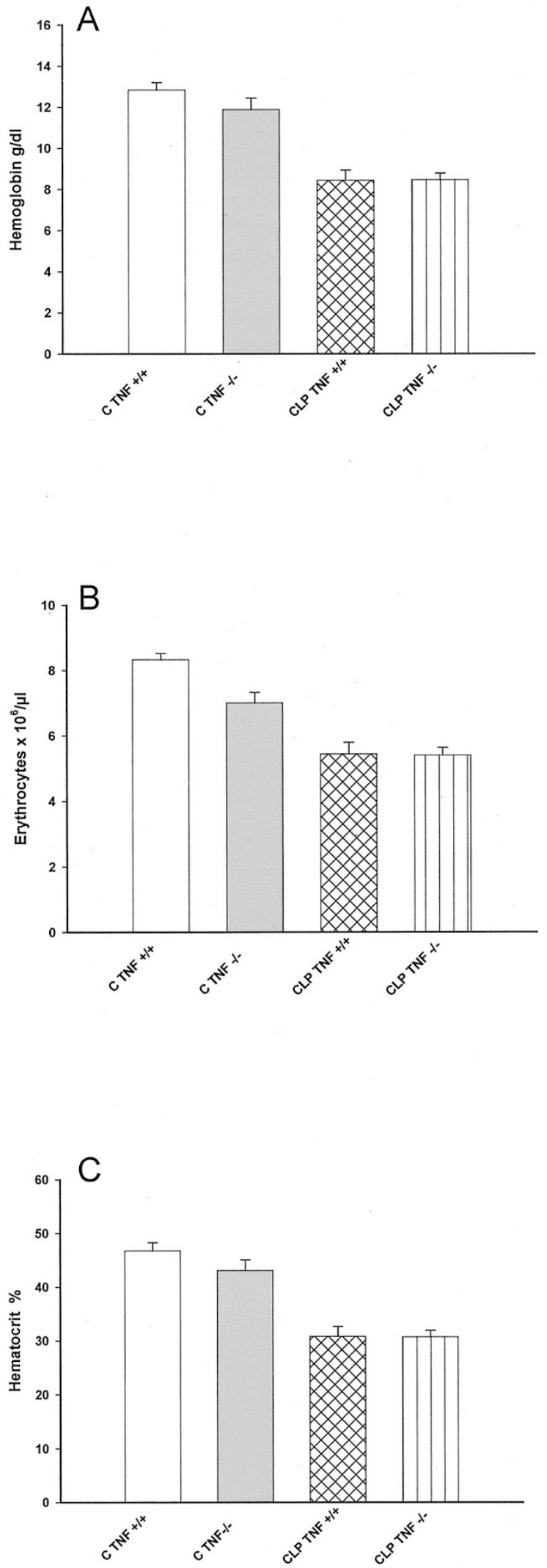

Figure 5.

TNF-deficient mice and wild-type mice equally developed anemia of chronic disease 2 weeks after cecal ligation and puncture (CLP). (A) Hemoglobin concentration, $(B)$ red blood cell count, and $(C)$ hematocrit were determined in wild-type and TNF-deficient mice 2 weeks after CLP. Results are given as mean $\pm \mathrm{SD}(n=5)$ regulation of TNF either systemically or in the bone marrow causes ACD.

In our newly established model of anemia induced by protracted inflammation after CLP, mice develop a microcytic, normochromic anemia with reduced serum iron and elevated iron stores, which is consistent with ACD. The reticulocyte counts drop considerably during the first week after CLP as a consequence of inhibited erythropoiesis in the bone marrow leading to the observed anemia. This acute depression of erythropoiesis in the bone marrow resembles the depression of erythropoiesis during systemic inflammation observed in septic patients. A slightly shortened halflife of erythrocytes during ACD has been reported for humans and in a rat model of ACD (Balfour Sartor et al, 1989). This may contribute to the generation of anemia in our model of ACD as well. ACD in our model is not mediated by a decrease of EPO levels. Anemia is most marked 2 weeks after CLP. In a rat model for ACD established by application of arthropathic peptidoglycan-polysaccharide polymers, the lowest hematocrit levels were observed 2 weeks after onset of inflammation as well (Balfour Sartor et al, 1989). The time course of ACD in our model with most marked anemia 2 weeks after CLP reflects the time course of systemic inflammation caused by CLP, which vanishes after 2 to 3 weeks. The same phenomenon can be observed in humans once the cause of ACD is removed. In summary, we characterized a new mouse model for ACD that fulfils all criteria needed to diagnose ACD in humans and that seems suitable to test molecules implicated in the generation of ACD for their indispensability in the disease process. A discrepancy of the model to human ACD is the early extramedullary hematopoiesis in spleen and liver of mice, which is not usually found in patients suffering from ACD.

In mice treated with anti-TNF and control mice, we observed an identical time course of reticulocyte numbers, indicating depression of erythropoiesis at the same time points and to the same degree. Accordingly, mice that are TNF- deficient due to a disrupted TNF gene develop anemia to the same extent as wild-type mice within 2 weeks after CLP. Iron serum concentrations and iron stores do not differ between TNF-deficient mice and wild-type mice after CLP.

Our findings are in contrast to the previously reported in vivo data suggesting a role of TNF in the generation of anemia. The above mentioned effects of TNF on iron metabolism and erythropoiesis in nude mice reported by Johnson et al (1989) were caused by a relatively high systemic TNF concentration of up to $68 \mathrm{ng} / \mathrm{ml}$ induced by application of TNF-secreting tumor cells. TNF serum concentrations in mice after CLP are only detectable after stimulation with bacterial lipopolysaccharide and range from 50 to $200 \mathrm{pg} / \mathrm{ml} 2$ days after CLP (Echtenacher et al, 2003). Furthermore, local TNF concentrations in the bone marrow during ACD are unknown. Therefore, it is difficult to estimate the significance of effects gained by high systemic concentrations of TNF as reported by Johnson et al (1989).

The use of TNF-deficient mice and of neutralizing antibodies to TNF in our murine model for ACD clearly 
A

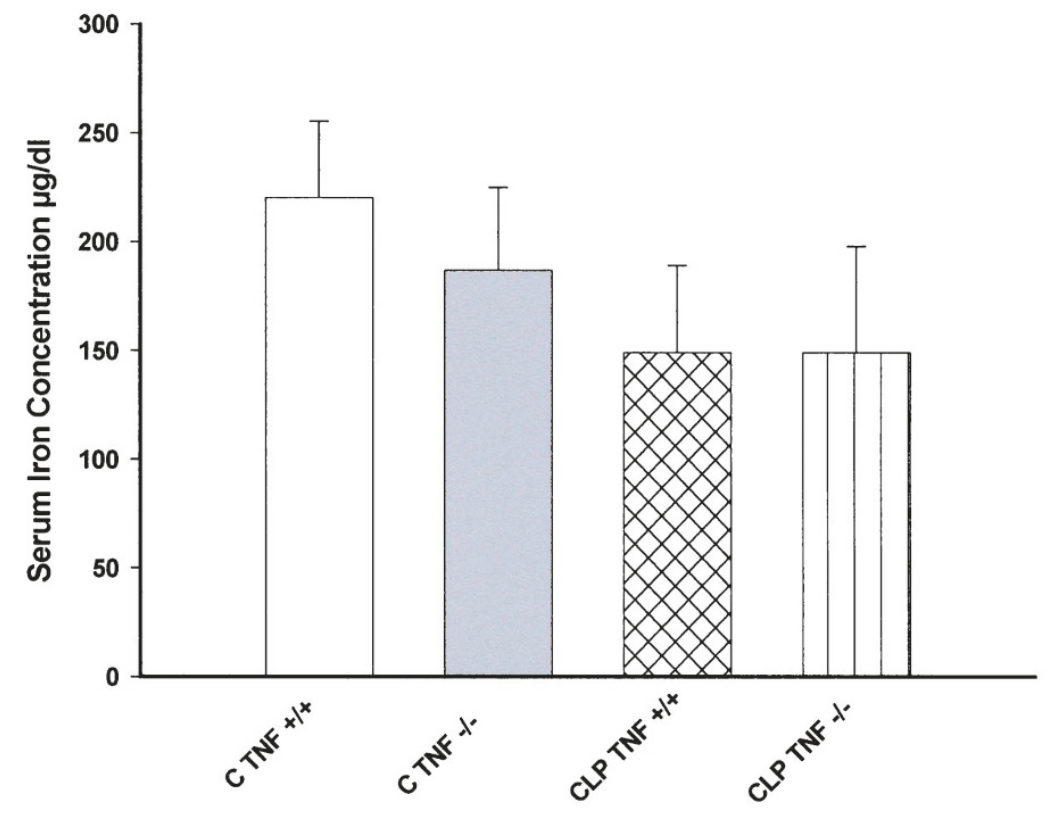

B

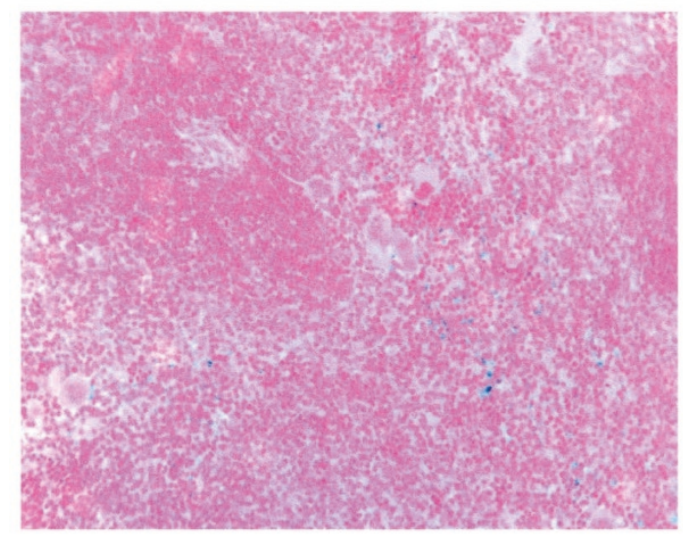

C -1-

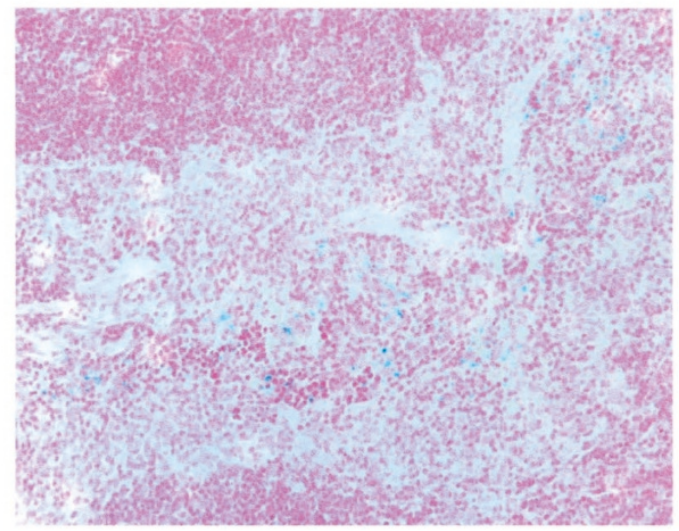

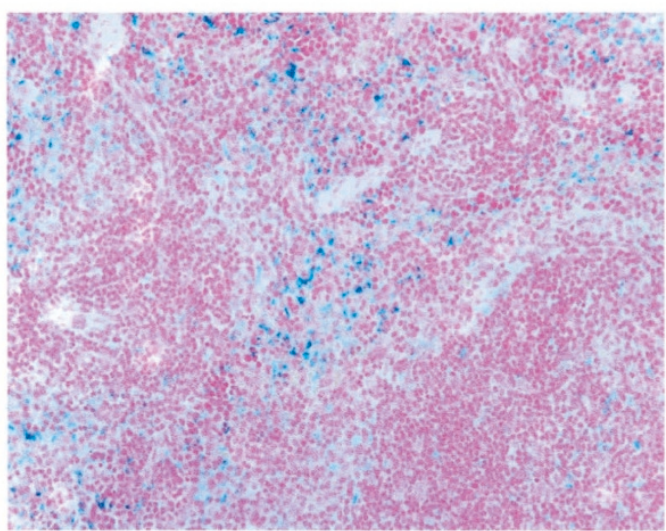

\section{CLP -/-}

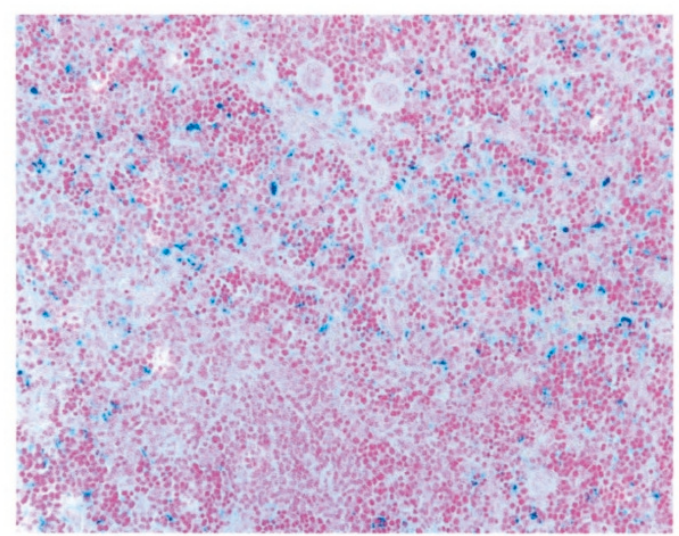

\section{Figure 6.}

Serum iron concentrations and iron stores in the spleen were determined in TNF-deficient and wild-type mice 2 weeks after cecal ligation and puncture (CLP). (A) Serum iron concentration in wild-type and TNF-deficient mice were determined 2 weeks after CLP. Results are given as mean \pm SD ( $n=5)$. (B) Iron-stained histologic section of splenic tissue $(\times 20)$ from untreated control mouse $(C+/+)$ and untreated TNF-deficient mouse $(C-/-)$, with only few iron-positive cells in the red pulp. Wild-type mouse $(\mathrm{CLP}+/+)$ and TNF-deficient mouse $(\mathrm{CLP}-/-) 2$ weeks after CLP had equally increased numbers of iron-positive cells in the red pulp. 
demonstrate that ACD can develop in the absence of TNF activity. Therefore, TNF is unlikely to be of major importance in the generation of ACD at least in the murine system. However, in the discussion of our findings, one has to keep in mind that ACD in humans is a heterogeneous disorder that can be induced by a number of different inflammatory or neoplastic processes, which also means that the relevant mechanism might differ depending on the specific underlying disease.

In contrast to our findings in the mouse model of ACD induced by severe systemic inflammation, two clinical studies in patients with rheumatoid arthritis showed that blockade of TNF by a chimeric mAb only induced a slight increase of blood hemoglobin concentrations (Davis et al, 1997; Papadakis et al, 2002). This might as well be explained by TNF effects not specifically directed toward erythropoietic cells but rather as a general down-regulation of proinflammatory cytokines downstream of TNF. Interestingly, similar effects were found after administration of oral prednisolone to patients suffering from rheumatoid arthritis and ACD (Kendall et al, 1993), supporting the notion that inhibition of production and action of mediators of inflammation can cause minor but significant increases in hemoglobin in ACD. Additionally, TNF blockade as well as oral prednisolone lead to a reduction of the EPO serum concentration (Davis et al, 1997; Kendall et al, 1993), ruling out that TNF negatively regulates EPO production.

To summarize, although there is a large body of in vitro data and clinical reports suggesting TNF as a mediator of ACD, the data from two crucial clinical studies in which neutralizing antibodies to TNF were used to correct $A C D$ are not suitable to classify a role of TNF as a major mediator of ACD. On the contrary, the finding reported by Papadakis et al (2002) that control individuals and patients with rheumatoid arthritis and iron-deficiency anemia showed increased hemoglobin values in a similar range as ACD patients with rheumatoid arthritis rather suggests that neutralization of TNF influences hematopoiesis by mechanisms that are not specific to ACD. Even though TNF has been discussed in the literature as a physiological negative regulator of erythropoiesis (Zhang et al, 1995), the published data do not allow one to draw conclusions concerning the mechanisms involved.

The results of this study clearly demonstrate that TNF is not crucial for induction of ACD in the model with transient anemia induced by protracted septic peritonitis. Taking into account our findings in the mouse model, the contribution of TNF to human ACD should be critically reevaluated.

\section{Materials and Methods}

\section{Mice}

Female or male NMRI mice (25 to $30 \mathrm{~g}$ ) were purchased from Charles River, (Sulzfeld, Germany). TNF-deficient mice were generated by Pasparakis et al (1996) and raised in Forschungszentrum für Umwelt und Gesundheit (GSF), Munich, and kindly provided by Lothar Hült- ner. Animals were kept under standard conditions at the animal facility of the University of Regensburg. Experiments were performed according to federal guidelines for animal experimentation. Animals in control groups and treated groups were age and sex matched.

\section{Cecal Ligation and Puncture}

Mice were anesthetized by ip injection of $75 \mathrm{mg} / \mathrm{kg}$ Ketanest (Parke, Davis \& Company, Munich, Germany) and $16 \mathrm{mg} / \mathrm{kg}$ Rompun (Bayer AG, Leverkusen, Germany). The cecum was exteriorized and the distal end was ligated and punctured to achieve a sublethal CLP as described previously (Echtenacher et al, 1990).

\section{Reagents}

Rat antimouse-TNF IgD was injected ip at a concentration of $4 \mathrm{mg} / \mathrm{kg}$ per body weight on Day 1 after CLP. The antibody and its neutralizing capacity was described in detail by Echtenacher et al (1990).

\section{Blood and Bone Marrow Parameters}

Red blood cell count, hemoglobin concentration, mean corpuscular volume, mean corpuscular hemoglobin concentration, and hematocrit were measured by the ADVIA120 hematology system (Bayer Diagnostics, Fernwald, Germany). Serum iron concentration was measured by the ADVIA1650 chemistry system (Bayer Diagnostics). For these hematologic determinations, groups of mice $(n=5)$ were anesthetized, and blood was drawn at the indicated time points and analyzed. Afterward the mice were killed by cervical dislocation. For serial reticulocyte counts, a droplet of blood was obtained after cutting off a small piece of the tip of the tail. Control animals that had undergone an identical bleeding regimen had not become anemic (data not shown). Blood smears were prepared immediately and stained with Brilliant Cresyl Blue (Fluka Chemie GmbH, Buchs, Switzerland) for 10 minutes in a moist chamber. Bone marrow cells were sampled from the femur by injecting and aspirating three times $1 \mathrm{ml}$ of FCS as washing medium using a syringe introduced into the bone cavity. Differentiation of bone marrow cells was pursued on MayGruenwald-Giemsa-stained smears.

\section{Sandwich ELISA for Detection of EPO}

Mouse EPO concentrations in serum were measured according to the method recently described by Chang and Stevenson (2002) using the commercially available antibodies described by the authors.

\section{Histology}

Mouse spleen and liver tissue was fixed in $4 \%$ buffered formalin overnight. Formalin-fixed tissue was dehydrated and embedded in paraffin, sectioned, and hematoxylin and eosin stained, or used to evaluate iron stores by Turnbull's acid ferrocyanide reaction. 


\section{Statistical Analysis}

All experiments were conducted at least three times. $P$ values were determined using the Student's $t$ test.

\section{References}

Akahane K, Hosoi T, Urabe A, Kawakami A, and Takaku F (1987). Effects of recombinant human tumor necrosis factor on normal and mouse hematopoietic progenitor cells. Int $\mathrm{J}$ Cell Clon 5:16-26.

Alvarez-Hernandez X, Liceaga J, McKay IC, and Brock JH (1989). Induction of hypoferremia and modulation of macrophage iron metabolism by tumor necrosis factor. Lab Invest 61:319-322.

Balfour Sartor R, Anderle SK, Rifai N, Goo D, Cromartie WJ, and Schwab JH (1989). Protracted anemia associated with chronic, relapsing systemic inflammation induced by arthropathic peptidoglycan-polysaccharide polymers in rats. Infect Immun 57:1177-1185.

Barrett-Connor E (1972). Anemia and infection. Am J Med 52:242-253.

Cartwright GE (1966). The anemia of chronic disorders. Semin Hematol 3:351-364.

Chang KH and Stevenson MM (2002). Comparison of murine Epo ELISA and Epo bioassays in detecting serum Epo levels during anemia associated with malaria infection. J Immunol Meth 262:129-136.

Davis D, Charles J, Potter A, Feldmann M, Maini RN, and Elliott MJ (1997). Anaemia of chronic disease in rheumatoid arthritis: In vivo effects of tumor necrosis factor alpha blockade. $\mathrm{Br} \mathrm{J}$ Rheumatol 36:950-956.

Echtenacher B, Falk W, Männel DN, and Krammer PH (1990). Requirement of endogenous tumor necrosis factor/cachectin for recovery from experimental peritonitis. J Immunol 145: 3762-3766.

Echtenacher B, Urbaschek R, Weigl K, Freudenberg MA, and Männel DN (In press, 2003). Treatment of experimental sepsis-induced immunoparalysis with TNF. Immunobiol.

Freireich EJ, Miller A, Emerson CP, and Ross JF (1957). The effect of inflammation on the utilization of erythrocyte and transferrin bound radioiron for red cell production. Blood 12:972-981.

Johnson CS, Cook CA, and Furmanski P (1990). In vivo suppression of erythropoiesis by tumor necrosis factor: Reversal with exogenous erythropoietin. Exp Hematol 18:109-113.

Johnson RA, Waddelow TA, Caro J, Oliff A, and Roodman GD (1989). Chronic exposure to tumor necrosis factor in vivo preferentially inhibits erythropoiesis in nude mice. Blood 74:130-138.

Jongen-Lavrencic M, Peeters HRM, Backx B, Touw IP, Vreugdenhil G, and Swaak AJG (1994). R-h-erythropoietin counteracts the inhibition of in vitro erythropoiesis by tumor necrosis factor alpha in patients with rheumatoid arthritis. Rheumatol Int 14:109-113.

Kendall R, Wasti A, Harvey A, Hill J, Chapman C, Norfolk DR, and Pullar T (1993). The relationship of haemoglobin to serum erythropoietin concentrations in the anaemia of rheumatoid arthritis: The effect of oral prednisolone. Rheumatology 32:204208.
Means RT Jr and Krantz SB (1993). Inhibition of human erythroid colony-forming units by tumor necrosis factor requires beta interferon. J Clin Invest 91:416-430.

Means RT Jr, Dessypris EN, and Krantz SB (1992). Inhibition of human erythroid colony-forming units by interleukin-1 is mediated by gamma interferon. J Cell Physiol 150:59-71.

Means RT Jr, Krantz SB, Luna J, Marsters SA, and Ashkenazi A (1994). Inhibition of murine erythroid colony-formation in vitro units by interferon gamma and correction by interferon receptor immunoadhesion. Blood 83:911-915.

Miller CB, Jones RJ, Piantadosi S, Abeloff MD, and Spivak JL (1990). Decreased erythropoietin response in patients with the anemia of cancer. N Engl J Med 322:1689-1692.

Moldawer LL, Marano MA, Wei H, Fong Y, Silen ML, Kuo G, Manogue KR, Vlassara $\mathrm{H}$, Cohen $\mathrm{H}$, and Cerami A (1989). Cachectin/tumor necrosis factor-alpha alters red blood cell kinetics and induces anemia in vivo. FASEB J 3: 1637-1643.

Papadakis HA, Kritikos HD, Valatas V, Boumpas D, and Eliopoulos GD (2002). Anemia of chronic disease in rheumatoid arthritis is associated with increased apoptosis of bone marrow erythroid cells: Improvement following anti-tumor necrosis factor- $\alpha$ antibody therapy. Blood 100:474-482.

Pasparakis M, Alexopoulou L, Episkopou V, and Kollias G (1996). Immune and inflammatory responses in TNF alphadeficient mice: A critical requirement for TNF alpha in the formation of primary B cell follicles, follicular dendritic cell networks and germinal centers, and in the maturation of the humoral immune response. J Exp Med 184:1207-1211.

Rogers JT, Bridges KR, Durmowicz GP, Glass J, Auron PE, and Munro HN (1990). Translational control during the acute phase response: Ferritin synthesis in response to interleukin-1. J Biol Chem 265:14572-14578.

Rusten LS and Jacobsen SEW (1995). Tumor necrosis factor directly inhibits human erythropoiesis in vitro: Role of p55 and p75 TNF receptors. Blood 85:989-996.

Salleri C, Maciejewski JP, Sato T, Young NS (1996). Interferon gamma constitutively expressed in the stromal microenvironment of human marrow cultures mediates potent hematopoietic inhibition. Blood 87:4149-4164.

Stockenhuber F, Keil M, Wurnig C, Kurz RW, GottsaunerWolf M, and Balcke P (1994). Impaired erythropoietin responsiveness in anaemic rheumatoid arthritis patients: Potential relation to immune mechanisms. Clin Sci 86:633-638.

Vreugdenhil G, Lowenberg B, Van Eijk HG, and Swaak AJ (1992). Tumor necrosis factor alpha is associated with disease activity and the degree of anemia in patients with rheumatoid arthritis. Eur J Clin Invest 22:488-493.

Wang CQ, Udupa KB, and Lipschitz DA (1995). Interferon gamma exerts its negative regulatory effect primarily on the earliest stages of murine erythroid progenitor cell development. J Cell Physiol 162:134-138.

Zhang Y, Harada A, Bluthmann H, Wang JB, Nakao S, Mukaida $\mathrm{N}$, and Matsushima K (1995). Tumor necrosis factor (TNF) is a physiologic regulator of hematopoietic progenitor cells: Increase of early hematopoietic progenitor cells in TNF receptor p55-deficient mice in vivo and potent inhibition of progenitor cell proliferation by TNF in vitro. Blood 86:2930-2937.

Zucker S, Friedman S, and Lysik RM (1974). Bone marrow erythropoiesis in the anemia of infection, inflammation, and malignancy. J Clin Invest 53:1132-1138. 